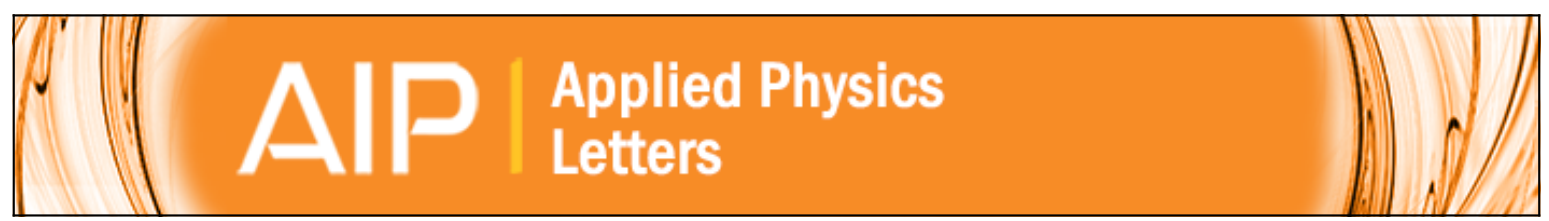

Lifetime recovery in ultrahighly titanium-doped silicon for the implementation of an intermediate band material

E. Antolín, A. Martí, J. Olea, D. Pastor, G. González-Díaz, I. Mártil, and A. Luque

Citation: Applied Physics Letters 94, 042115 (2009); doi: 10.1063/1.3077202

View online: $\mathrm{http} / / / \mathrm{dx}$. doi.org/10.1063/1.3077202

View Table of Contents: http://scitation.aip.org/content/aip/journal/apl/94/4?ver=pdfcov

Published by the AIP Publishing

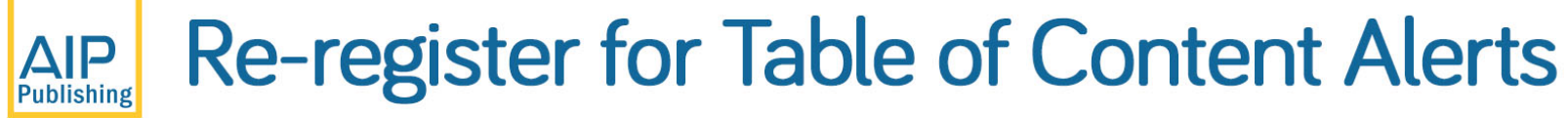

Create a profile. 


\title{
Lifetime recovery in ultrahighly titanium-doped silicon for the implementation of an intermediate band material
}

\author{
E. Antolín, ${ }^{1, a)}$ A. Martí, ${ }^{1}$ J. Olea, ${ }^{2}$ D. Pastor, ${ }^{2}$ G. González-Díaz, ${ }^{2}$ I. Mártil, ${ }^{2}$ and A. Luque ${ }^{1}$ \\ ${ }^{1}$ Instituto de Energía Solar, E.T.S.I. Telecomunicación, Universidad Politécnica de Madrid, \\ 28040 Madrid, Spain \\ ${ }^{2}$ Dpto. de Física Aplicada III (Electricidad y Electrónica), Facultad de Ciencias Físicas, \\ Universidad Complutense de Madrid, 28040 Madrid, Spain
}

(Received 2 November 2008; accepted 12 January 2009; published online 30 January 2009)

\begin{abstract}
The doping of conventional semiconductors with deep level (DL) centers has been proposed to synthesize intermediate band materials. A recent fundamental study of the nonradiative recombination (NRR) mechanisms predicts the suppression of the NRR for ultrahigh DL dilutions as a result of the delocalization of the impurity electron wave functions. Carrier lifetime measurements on $\mathrm{Si}$ wafers doped with $\mathrm{Ti}$ in the $10^{20}-10^{21} \mathrm{~cm}^{-3}$ concentration range show an increase in the lifetime, in agreement with the NRR suppression predicted and contrary to the classic understanding of DL action. (C) 2009 American Institute of Physics. [DOI: 10.1063/1.3077202]
\end{abstract}

It was determined long ago ${ }^{1}$ that the presence of deep level (DL) impurities, which gives rise to the so-called Shockley-Read-Hall (SRH) recombination, ${ }^{2,3}$ constitutes one of the main causes of recombination in semiconductors. Consequently, a great technological effort has been devoted to producing impurity-free semiconductors in order to obtain long carrier lifetimes in manufactured devices. However, the proposal of the intermediate band solar cell (IBSC), ${ }^{4}$ a photovoltaic device with potential for very high efficiencies, has prompted the question as to whether it is possible to take advantage of the optical transitions introduced by DLs without suffering a strong nonradiative recombination (NRR).

The IBSC operation is based on the use of a material that possesses an electronic energy band of allowed states (intermediate band, IB) within the conventional bandgap (a detailed discussion of the IBSC operating principles can be found in Refs. 4 and 5). Although all practical IBSC prototypes reported to date have been manufactured using quantum dot arrays, ${ }^{6}$ there is a practical interest in implementing bulk IB materials. In this respect, promising results have been obtained with highly mismatched alloys based on the band anticrossing mechanism. ${ }^{7}$ It has also been suggested that any element that produces DLs in a given semiconductor can be used, in principle, to manufacture an IB material with suppressed NRR. ${ }^{8}$ This assessment is based on a fundamental study of the NRR mechanisms, which has led to results with application probably beyond the IBSC context. The model presented in Ref. 8 shows that the nonradiative energy transfer can be suppressed in DL-doped materials if the wave functions of the electrons trapped in the impurities become delocalized, as they are in the conduction and valence bands. This can occur if the impurity density is increased until the impurities are close enough to interact among themselves. Within this context, a delocalization by means of an Anderson transition ${ }^{9}$ is expected for concentrations of $1-2$ $\times 10^{20} \mathrm{~cm}^{-3}$ and a delocalization by means of a Mott transition ${ }^{10}$ for concentrations above $5.9 \times 10^{19} \mathrm{~cm}^{-3}$ at 300 $\mathrm{K}$. In this work, the prediction of NRR suppression is tested

\footnotetext{
a) Author to whom correspondence should be addressed. Electronic mail: elisa@ies-def.upm.es.
}

experimentally through lifetime measurements on $\mathrm{Si}$ wafers doped at ultrahigh concentrations with $\mathrm{Ti}$, a well-known lifetime killer for $\mathrm{Si}^{11}{ }^{11}$ As will be shown below, the results indicate that the peak concentrations achieved $(>2$ $\times 10^{20} \mathrm{~cm}^{-3}$ ) are above the actual critical concentration.

The samples, $300 \mu \mathrm{m}$ thick Si (111) wafers of high resistivity $\left(200 \Omega \mathrm{cm}\right.$, light $n$-doping $\left.N_{D}=2.2 \times 10^{13} \mathrm{~cm}^{-3}\right)$, have been implanted with Ti ions in ultrahigh doses $\left(10^{15}\right.$, $5 \times 10^{15}$, and $10^{16} \mathrm{~cm}^{-2}$ ) and with different energies in the range of $20-35 \mathrm{keV}$. Since the reported solubility values of Si:Ti using conventional annealing methods ${ }^{12,13}$ are much lower than the predicted critical concentration, the pulsed laser melting (PLM) method ${ }^{14}$ has been applied to recover the crystallinity of the implanted layers. It consists of irradiating the wafers with a high energy laser pulse to melt the damaged layer for an interval of several nanoseconds and let it recrystallize using the underlying material as a seed. $\mathrm{A} \mathrm{KrF}$ excimer laser $(\lambda=248 \mathrm{~nm})$ was used with energy densities between 0.2 and $0.8 \mathrm{~J} / \mathrm{cm}^{2}$ and pulse width of $20 \mathrm{~ns}$, annealing with one or two pulses. Channeling Rutherford backscattering measurements showed recrystallization for the energy density of $0.6 \mathrm{~J} / \mathrm{cm}^{2}$ or higher, in agreement with previous studies on $\mathrm{KrF}$ laser melting effect on $\mathrm{Si}^{15}{ }^{15}$ Further details on the crystalline characterization are given in Ref. 16.

Table I shows the total Ti content deduced from time of flight secondary ion mass spectroscopy (Tof-SIMS) measurements in a series of samples that have been PLM annealed

TABLE I. Ti concentration in samples implanted with a dose of $10^{16} \mathrm{~cm}^{-2}$ that were PLM annealed with different laser energy densities or number of pulses.

\begin{tabular}{ccc}
\hline \hline $\begin{array}{c}\text { Annealing energy density } \\
\left(\mathrm{J} / \mathrm{cm}^{2}\right)\end{array}$ & Number of pulses & $\begin{array}{c}\text { Ti areal density } \\
\left(\mathrm{cm}^{-2}\right)\end{array}$ \\
\hline As-implanted & 0 & $1.00 \times 10^{16}$ \\
0.2 & 1 & $8.54 \times 10^{15}$ \\
0.4 & 1 & $8.45 \times 10^{15}$ \\
0.6 & 1 & $7.73 \times 10^{15}$ \\
0.6 & 2 & $7.31 \times 10^{15}$ \\
\hline \hline
\end{tabular}




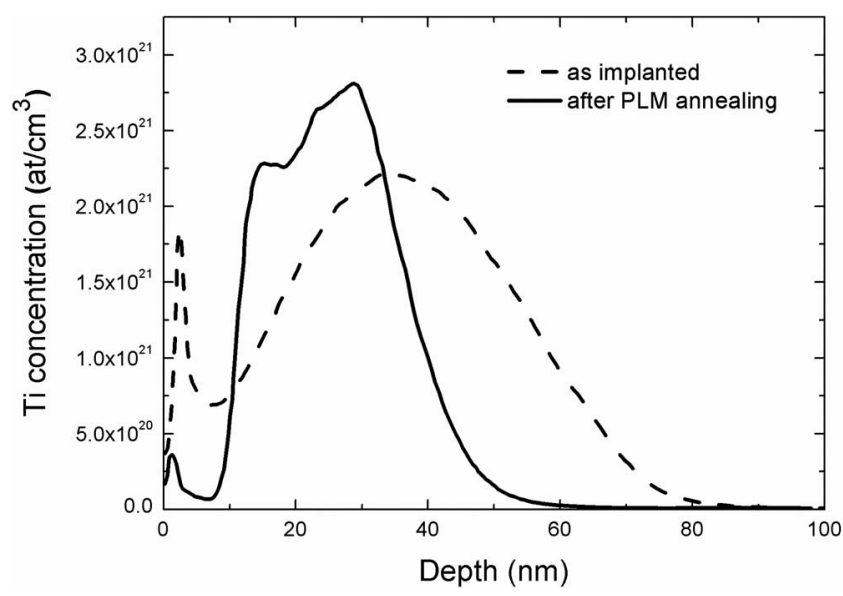

FIG. 1. Ti concentration profile of the implanted layer from SIMS measurements. The dashed line corresponds to an as-implanted sample and the solid line to a sample annealed with two laser pulses of $0.6 \mathrm{~J} / \mathrm{cm}^{2}$.

with different laser energy densities or number of pulses. All of them had been originally implanted with $35 \mathrm{keV}$ and a dose of $10^{16} \mathrm{~cm}^{-2}$. It can be seen that the amount of Ti lost through the surface increases when the energy density used in the annealing is higher, but it is still lower than 30\% in the sample that suffered the strongest annealing (two pulses of $0.6 \mathrm{~J} / \mathrm{cm}^{2}$ ). The relatively high amount of Ti that remains in the lattice corroborates the validity of the PLM method to obtain $\mathrm{Ti}$ densities in Si above the solubility limit.

Figure 1 shows the $\mathrm{Ti}$ concentration profiles of two samples as extracted from SIMS measurements. Both samples were implanted with $35 \mathrm{keV}$ and a dose of $10^{16} \mathrm{~cm}^{-2}$. One of them was not annealed (dashed line) and the other one (solid line) was annealed with two laser pulses of $0.6 \mathrm{~J} / \mathrm{cm}^{2}$. Disregarding surface transient artifacts of the SIMS measurement, the as-implanted sample shows a Gaussian profile, peaking with approximately 2.2 $\times 10^{21} \mathrm{~cm}^{-3}$. In the annealed sample there has been a migration of $\mathrm{Ti}$ atoms to the melted layer during the recrystallization, leading to a higher volumetric doping (between 2.3 and $2.8 \times 10^{21} \mathrm{~cm}^{-3}$ ). It must be noted that the resulting profile has a sharper form, which is more suitable for our purposes, since Gaussian tails are regions in which the Ti concentration, although high, is still below the delocalization transition point, and therefore, they behave potentially as highly SRHrecombining regions.

Figure 2 shows the effective wafer carrier lifetime $\tau_{\text {eff }}$ for different injection levels $\Delta n$ measured in Si samples that have been implanted with different ultrahigh doses of Ti [in (a) the light was impinging on the implanted layer and in (b) the wafers were measured with the light entering through the back of the sample]. The measurements have been taken using the quasi-steady-state photoconductance technique. ${ }^{17}$ This is a contactless technique. An impedance bridge inductively coupled to the sample is used to measure the changes in conductivity produced by illumination with a slow white light flash. The samples have been chosen so that a higher doping is accompanied by a higher implantation energy $\left(25,30\right.$, and $35 \mathrm{keV}$ for the samples implanted with $10^{15}$, $5 \times 10^{15}$, and $10^{16} \mathrm{~cm}^{-2} \mathrm{Ti}$ atoms, respectively). They were all annealed with two laser pulses of $0.6 \mathrm{~J} / \mathrm{cm}^{2}$.

To interpret these plots it must be taken into account that the white light of the flash is absorbed through the whole
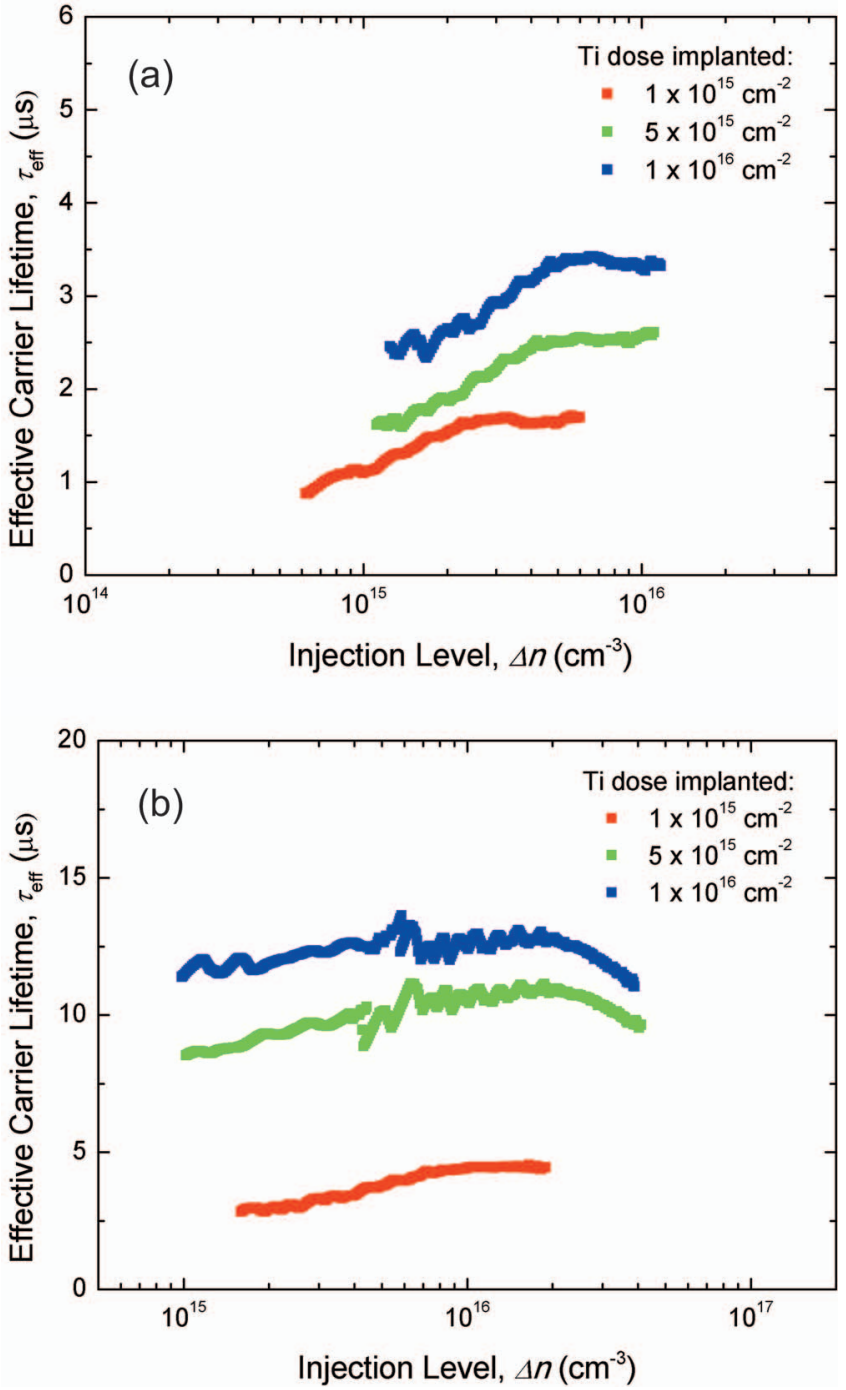

FIG. 2. (Color) Wafer effective carrier lifetime vs carrier injection level in samples implanted with Ti doses of $10^{15}, 5 \times 10^{15}$, and $10^{16} \mathrm{~cm}^{-2}$. (a) Measured with the implanted layer located at the surface impinged by the flash light. (b) Measured with the back surface of the wafer impinged by the flash light.

wafer thickness. Thus, the data presented correspond to the effective wafer lifetime, which is a function of the bulk lifetime (i.e., the lifetime in the unaltered $\mathrm{Si}$ substrate, in our case $200 \mu$ s for the interval between $10^{14}$ and $10^{18} \mathrm{~cm}^{-3}$ carriers injected), the implanted layer lifetime, and the surface recombination (not significant here since the wafers were submerged in $\mathrm{HF}: \mathrm{H}_{2} \mathrm{O}$ to passivate the surfaces during the measurements $\left.{ }^{18}\right)$. Both the effective wafer lifetime and the injection level $\Delta n$ (the average excess carrier density produced by light generation) were calculated by means of the continuity equation from the conductance of the sample measured under different calibrated illumination densities. ${ }^{17}$ As the measurement is taken in a quasi-steady-state, the maximum injection level achieved for a sample is limited by its effective wafer lifetime. In the cases of higher effective wafer lifetime [samples with doses $5 \times 10^{15}$ and $10^{16} \mathrm{~cm}^{-2}$ in Fig. 2(b)], the maximum injection with the flash lamp used is just high enough $\left(>2 \times 10^{16} \mathrm{~cm}^{-3}\right)$ to see the onset of the lifetime drop related to a rise of the Auger recombination rate, typical of $\mathrm{Si}$ samples. ${ }^{19}$

It can be seen in both plots that the effective lifetimes measured show an increase with the Ti dose implanted. The 
tendency of a lifetime recovery with Ti concentration agrees with the prediction of the NRR suppression when the DL impurity exceeds the delocalization transition. ${ }^{8}$ On the other hand, the effective wafer lifetime obtained for all implanted samples is lower than that of the unaltered $\mathrm{Si}$ substrate. This can be attributed to the existence of doping tails. Even though the PLM annealing sharpens the concentration profile (see Fig. 1), there is still a region with a high amount of Ti but where the concentration is too low to reach the delocalization transition. In these regions SRH recombination is necessarily very strong. This fact may constitute a drawback of the preparation method for the implementation of practical IB materials. Another reason for the lifetime drop can be an insufficient recovery of the crystalline quality. It has been reported about polycrystallinity or implantation-induced dislocation loops that spread below the doped layer in highly implanted Si samples annealed with KrF laser. ${ }^{15}$ In agreement with that reference, glancing incident $\mathrm{x}$-ray diffraction characterization of our samples has revealed the existence of polycrystalline phases after laser annealing, only observable for very high implantation doses $\left(10^{16} \mathrm{~cm}^{-2}\right){ }^{16}$ In this respect, there is probably room for improvement through the optimization of the annealing procedure in future samples. It must be noted, however, that both explanations for the lifetime drop, the existence of doping tails and damage of the crystalline lattice, imply a more severe degradation in the lifetime for higher implantation doses. The fact that an increment in the lifetime for higher doses is observable in Fig. 2 indicates that the mechanism that induces a lifetime recovery for high concentrations, which we identify with the NRR recombination suppression predicted in, ${ }^{8}$ has to be significant enough to counteract, to a large extent, these nonidealities.

In the analysis of photoconductance data, the effective lifetime is obtained under the assumption of homogeneous carrier excitation in the wafer. This is the standard method used for Si samples because, even though most of the luminous excitation is produced in the first tens of microns, the excitation is transferred almost homogeneously across the whole wafer as a result of the high bulk lifetime and carrier mobility. That is not the case in our samples, in which the doped layer has a much lower lifetime than the bulk. Thus, when the wafer is illuminated at the rear side, the obtained wafer $\tau_{\text {eff }}$ is slightly higher than that under front illumination. The comparison between Figs. 2(a) and 2(b) agrees with this argument and corroborates that the small differences presented are not a measuring artifact (caused for instance by different reflection of light on wafers with different implants).
In summary, ultrahighly Ti-doped $\mathrm{Si}$ material, with a $\mathrm{Ti}$ concentration far above the solid solubility limit, was obtained through the implantation and subsequent PLM annealing of the samples. Carrier lifetime measurements indicate that in the range between $10^{15}$ and $10^{16} \mathrm{~cm}^{-2}$ of $\mathrm{Ti}$ atoms implanted, which lies above the delocalization transition impurity concentration, the effective lifetime of the wafers increases with Ti concentration. This result is contrary to the classic understanding of the DL impurity action in semiconductors and supports the predictions ${ }^{8}$ made about a NRR suppression for the ultrahigh doping of semiconductors with DLs. It also illustrates the conditions necessary to produce successful IB solar cells based on semiconductor materials doped with DL impurities.

This work has been supported by the project FULLSPECTRUM (Grant No. SES6-CT-2003-502620) funded by the European Commission, by the Regional Government of Madrid within the project NUMANCIA (Grant No. S-0505/ ENE/000310), and by the Spanish National Research Program within the project GENESIS-FV (Grant No. CSD20060004).

${ }^{1}$ F. Seitz, Trans. Faraday Soc. 35, 74 (1939).

${ }^{2}$ W. Shockley and W. T. Read, Phys. Rev. 87, 835 (1952).

${ }^{3}$ R. N. Hall, Phys. Rev. 87, 387 (1952).

${ }^{4}$ A. Luque and A. Martí, Phys. Rev. Lett. 78, 5014 (1997).

${ }^{5}$ A. Martí, L. Cuadra, and A. Luque, in Next Generation Photovoltaics: High Efficiency through Full Spectrum Utilization, edited by A. Martí and A. Luque (Institute of Physics Publishing, Bristol, 2003), pp. 140-162.

${ }^{6}$ A. Martí, L. Cuadra, and A. Luque, Conference Record of the 28th IEEE Photovoltaics Specialists Conference, 2000 (unpublished), pp. 940-943.

${ }^{7}$ W. Walukiewicz, W. Shan, K. M. Yu, J. W. Ager, E. E. Haller, I. Miotkowski, M. J. Seong, H. Alawadhi, and A. K. Ramdas, Phys. Rev. Lett. 85, 1552 (2000).

${ }^{8}$ A. Luque, A. Martí, E. Antolín, and C. Tablero, Physica B 382, 320 (2006).

${ }^{9}$ P. W. Anderson, Phys. Rev. 109, 1492 (1958).

${ }^{10}$ N. F. Mott, Rev. Mod. Phys. 40, 677 (1968).

${ }^{11}$ A. Rohatgi, J. R. Davis, R. H. Hopkins, P. Rai-Choudhury, P. G McMullin, and J. R. McCormick, Solid-State Electron. 23, 415 (1980).

${ }^{12}$ S. Hocine and D. Mathiot, Appl. Phys. Lett. 53, 1269 (1988).

${ }^{13}$ D. Mathiot and D. Barbier, J. Appl. Phys. 69, 3878 (1991).

${ }^{14}$ C. W. White, S. R. Wilson, B. R. Appleton, and F. W. Young, Jr., J. Appl. Phys. 51, 738 (1980)

${ }^{15}$ J. Narayan, C. W. White, M. J. Aziz, B. Strizker, and A. Walthuis, J. Appl. Phys. 57, 564 (1985).

${ }^{16}$ J. Olea, M. Toledano-Luque, D. Pastor, G. González-Díaz, and I. Mártil, J. Appl. Phys. 104, 016105 (2008).

${ }^{17}$ R. A. Sinton and A. Cuevas, Appl. Phys. Lett. 69, 2510 (1996).

${ }^{18}$ R. Lago-Aurrekoetxea, I. Tobías, C. del Cañizo, and A. Luque, J. Electrochem. Soc. 148, G200 (2001).

${ }^{19}$ R. A. Sinton and R. M. Swanson, IEEE Trans. Electron Devices 34, 1380 (1987). 